\title{
Improving AOR Method for a Class of Two-by-Two Linear Systems
}

\author{
Cuixia $\mathrm{Li}^{1}$, Shiliang $\mathrm{Wu}^{2}$ \\ ${ }^{1}$ College of Mathematics, Chengdu University of Infromation Technology, Chengdu, China \\ ${ }^{2}$ School of Mathematics and Statistics, Anyang Normal University, Anyang, China \\ E-mail: lixiatk@126.com,wushiliang1999@126.com,slwu@aynu.edu.cn \\ Received October 18, 2010; revised December 10, 2010; accepted December 15, 2010
}

\begin{abstract}
In this paper, the preconditioned accelerated overrelaxation (AOR) method for solving a class of two-by-two linear systems is presented. A new preconditioner is proposed according to the idea of [1] by Wu and Huang. The spectral radii of the iteration matrix of the preconditioned and the original methods are compared. The comparison results show that the convergence rate of the preconditioned AOR methods is indeed better than that of the original AOR methods, whenever the original AOR methods are convergent under certain conditions. Finally, a numerical example is presented to confirm our results.
\end{abstract}

Keywords: Preconditioner, AOR Method, Convergence, Comparison

\section{Introduction}

Sometimes we have to solve the following linear systems

$$
H x=f,
$$

where

$$
H=\left[\begin{array}{cc}
I-B_{1} & D \\
C & I-B_{2}
\end{array}\right]
$$

is non-singular with

$$
\begin{aligned}
& B_{1}=\left(b_{i j}\right)_{p \times p}, B_{2}=\left(b_{i j}\right)_{(n-p) \times(n-p)}, \\
& C=\left(c_{i j}\right)_{(n-p) \times p}, D=\left(d_{i j}\right)_{p \times(n-p)} .
\end{aligned}
$$

Systems such as (1.1) are important and appear in many different applications of scientific computing. For example, (1.1) are usually faced when the following generalized linear-squares problem is considered

$$
\min _{x \in \mathbb{R}^{n}}(A x-b)^{T} W^{-1}(A x-b),
$$

where $W$ is the variance-covariance matrix. One can see [2-5] for details.

As is known, the linear systems (1.1) can be solved by direct methods or iterative methods. Direct methods are widely employed when the order of the coefficient matrix $H$ is not too large, and are often regarded as robust methods. The memory and the computational require- ments for solving the large linear systems may seriously challenge the most efficient direct methods available today. The alternative is to use iterative methods established for solving the large linear systems. Naturally, it is necessary that we make the use of iterative methods instead of direct methods to solve the large sparse linear systems. Meanwhile, iterative methods are easier to implement efficiently on high performance computers than direct methods.

As is known, there exist three well-known classical iterative methods, i.e., Jacobi, Gauss-Seidel and successive overrelaxation (SSOR) method, which were fully covered in the excellent books by Varge [6] and Young [7]. To make the convergence rate of SSOR method better, accelerated overrelaxation (AOR) method was proposed in [8] by Hadjidimos.

To solve the linear systems (1.1) with the AOR iterative method, based on the structure of the matrix $H$, the matrix $H$ is split as follows

$$
H=I-\left[\begin{array}{cc}
0 & 0 \\
-C & 0
\end{array}\right]-\left[\begin{array}{cc}
B_{1} & -D \\
0 & B_{2}
\end{array}\right] .
$$

The AOR iterative method for solving (1.1) is established as follows

$$
x^{(i+1)}=T_{w, r} x^{(i)}+w g, i=0,1, \cdots
$$

where $w \neq 0, T_{w, r}$ is iteration matrix and is of the following form 


$$
\begin{aligned}
T_{w, r} & =\left[\begin{array}{cc}
I & 0 \\
r C & I
\end{array}\right]^{-1}\left\{(1-w) I+\left[\begin{array}{cc}
0 & 0 \\
-C & 0
\end{array}\right]+w\left[\begin{array}{cc}
B_{1} & -D \\
0 & B_{2}
\end{array}\right]\right\} \\
& =\left[\begin{array}{cc}
(1-w) I+w B_{1} & -w D \\
w(r-1) C-w r C B_{1} & (1-w) I+w B_{2}+w r C D
\end{array}\right]
\end{aligned}
$$

and

$$
g=\left[\begin{array}{cc}
I & 0 \\
-r C & I
\end{array}\right] f .
$$

Obviously, if $w=r$, then the AOR method reduces to the SOR method.

The spectral radii of the iteration matrix is decisive for the convergence and stability of the method, and the smaller it is, the faster the method converges when the spectral radii is smaller than 1 . To accelerate the convergence rate of the iterative method solving the linear systems (1.1), preconditioned methods are often used. That is,

$$
P H x=P f,
$$

where the preconditioner $P$ is a non-singular matrix.

If the matrix $P H$ is expressed as

$$
P H=\left[\begin{array}{cc}
I-B_{1}^{*} & D^{*} \\
C^{*} & I-B_{2}^{*}
\end{array}\right],
$$

then the preconditioned AOR method can be defined by

$$
x^{(i+1)}=T_{w, r}^{*} x^{(i)}+w g^{*},(w \neq 0), i=0,1, \cdots
$$

where

$$
T_{w, r}^{*}=\left[\begin{array}{cc}
(1-w) I+w B_{1}^{*} & -w D^{*} \\
w(r-1) C^{*}-w r C^{*} B_{1}^{*} & (1-w) I+w B_{2}^{*}+w r C^{*} D^{*}
\end{array}\right]
$$

and

$$
g^{*}=\left[\begin{array}{cc}
I & 0 \\
-r C^{*} & I
\end{array}\right] P f .
$$

In this paper, according to the idea of [1] by $\mathrm{Wu}$ and Huang, a new preconditioner is proposed to improve the convergence rate of the AOR method. Be similar to the work of [1] and [9], we compare the spectral radii of the iteration matrix of the preconditioned and the original methods. The comparison results show that the convergence rate of the preconditioned AOR methods is indeed superior to that of the original AOR methods, whenever the original AOR methods are convergent (to see the next section).

For convenience, we shall now briefly explain some of the terminology and lemmas. Let $C=\left(c_{i j}\right) \in \mathbb{R}^{n \times n}$ be an $n \times n$ real matrix. By $\operatorname{diag}(C)$, we denote the $n \times n$ diagonal matix coinciding in its diagonal with $c_{i i}$. For $A=\left(a_{i j}\right), B=\left(b_{i j}\right) \in \mathbb{R}^{n \times n}$, we write $A \geq B$ if $a_{i j} \geq b_{i j}$ holds for all $i, j=1,2, \cdots, n$. Calling $A$ is nonnegative if $A \geq 0,\left(a_{i j} \geq 0 ; i, j=1,2, \cdots, n\right)$, we say that $A-B \geq 0$ if and only if $A \geq B$ These definitions carry immediately over to vectors by identifying them with $n \times 1$ matrices. $\rho(\cdot)$ denotes the spectral radius of a matrix.

Lemma 1.1 [6] Let $A \in \mathbb{R}^{n \times n}$ be a nonnegative and irreducible $n \times n$ matrix. Then

1) A has a positive real eigenvalue equal to its spectral radius $\rho(A)$;

2) for $\rho(A)$, there corresponds an eigenvector $x>0$;

3) $\rho(A)$ is a simple eigenvalue of $A$.

4) $\rho(A)$ increases when any entry of $A$ increases.

Lemma 1.2 [10] Let $A$ be a nonnegative matrix. Then

1) If $\alpha x \leq A x$ for some nonnegative vector $x$, $x \neq 0$, then $\alpha \leq \rho(A)$.

2) If $A x \leq \beta x$ for some positive vector $x$, then $\rho(A) \leq \beta$. Moreover, if $A$ is irreducible and if $0 \neq \alpha x \leq A x \leq \beta x$ for some nonnegative vector $x$, then

$$
\alpha \leq \rho(A) \leq \beta
$$

and $x$ is a positive vector.

The outline of this paper is as follows. In Section 2, the spectral radii of the iteration matrix of the original and the preconditioned methods are compared. In Section 3, a numerical example is presented to illustrated our results.

\section{Preconditioned AOR Methods and Comparisons}

Now, let us consider the preconditioned linear systems,

$$
\tilde{H} x=\tilde{f},
$$

where $\tilde{H}=(I+\tilde{S}) H$ and $\tilde{f}=(I+\tilde{S}) f$ with

$$
\tilde{S}=\left[\begin{array}{ll}
S & 0 \\
0 & 0
\end{array}\right] .
$$

According to [1], here $S$ is taken as as follows

$$
S=\left[\begin{array}{ccccc}
0 & b_{12} & 0 & \cdots & 0 \\
0 & 0 & b_{23} & \cdots & 0 \\
\vdots & \vdots & \vdots & \cdots & \vdots \\
0 & 0 & 0 & \cdots & b_{p-1, p} \\
b_{p, 1} & 0 & 0 & \cdots & 0
\end{array}\right]_{p \times p}
$$

Naturally, we assume that there at least exists a nonzero number in the elements of $S$.

By simple computations, we obtain

$$
\tilde{H}=\left[\begin{array}{cc}
I-\left[B_{1}-S\left(I-B_{1}\right)\right] & (I+S) D \\
C & I-B_{2}
\end{array}\right]
$$


with

$$
\begin{aligned}
& B_{1}-S\left(I-B_{1}\right) \\
& =\left[\begin{array}{cccc}
b_{11}+b_{12} b_{21} & b_{12} b_{22} & \cdots & b_{1 p}+b_{12} b_{2 p} \\
b_{21}+b_{23} b_{31} & b_{22}+b_{23} b_{32} & \cdots & b_{2 p}+b_{23} b_{3 p} \\
\vdots & \vdots & \ddots & \vdots \\
b_{p 1} b_{11} & b_{p 2}+b_{p 1} b_{12} & \cdots & b_{p p}+b_{p 1} b_{1 p}
\end{array}\right] .
\end{aligned}
$$

Be similar to (1.2), $\tilde{H}$ can be expressed as

$$
\tilde{H}=I-\left[\begin{array}{cc}
0 & 0 \\
-C & 0
\end{array}\right]-\left[\begin{array}{cc}
B_{1}-S\left(I-B_{1}\right) & -(I+S) D \\
0 & B_{2}
\end{array}\right] .
$$

Then the preconditioned AOR method for (2.1) is defined as follows:

$$
x^{(i+1)}=\tilde{T}_{w, r} x^{(i)}+w \tilde{g},(w \neq 0), i=0,1, \cdots
$$

where

$$
\begin{aligned}
\tilde{T}_{w, r}= & {\left[(1-w) I+w\left[B_{1}-S\left(I-B_{1}\right)\right] w(r-1) C\right.} \\
& -w r C\left[B_{1}-S\left(I-B_{1}\right)\right]-w(I+S) D(1-w) I \\
& \left.+w B_{2}+w r C(I+S) D\right]
\end{aligned}
$$

and

$$
\tilde{g}=\left[\begin{array}{cc}
I & 0 \\
-r C & I
\end{array}\right] \tilde{f} .
$$

The following theorem is given by comparing the spectral radii of the iteration matrix $\tilde{T}_{w, r}$ and the original iteration matrix $T_{w, r}$.

Theorem 2.1 Let the coefficient matrix $H$ be irreducible, $B_{1} \geq 0$ with $\operatorname{diag}\left(B_{1}\right)>0, B_{2} \geq 0, C \leq 0$, $D \leq 0,0<w \leq 1$ and $0 \leq r<1$. Then

1) $\rho\left(\tilde{T}_{w, r}\right)<\rho\left(T_{w, r}\right)$, if $\rho\left(T_{w, r}\right)<1$;

2) $\rho\left(\tilde{T}_{w, r}\right)>\rho\left(T_{w, r}\right)$, if $\rho\left(T_{w, r}\right)>1$.

Proof. By simple computations, we obtain

$$
\begin{aligned}
T_{w, r}= & {\left[\begin{array}{cc}
(1-w) I+w B_{1} & -w D \\
w(r-1) C & (1-w) I+w B_{2}
\end{array}\right] } \\
& +w r\left[\begin{array}{cc}
0 & 0 \\
-C B_{1} & C D
\end{array}\right]
\end{aligned}
$$

Clearly, if the matrix $H$ satisfies $B_{1} \geq 0, B_{2} \geq 0$, $C \leq 0$ and $D \leq 0$ with $0<w \leq 1$ and $0 \leq r<1$, then

$$
\left[\begin{array}{cc}
0 & 0 \\
-C B_{1} & C D
\end{array}\right] \geq 0 \text { and } T_{w, r} \geq 0 .
$$

Since the matrix $H$ is irreducible, by observing the structure of (2.3), it is not difficult to get that the matrix $T_{w, r}$ is irreducible. Similarly, the matrix $\tilde{T}_{w, r}$ is nonnegative and irreducible with $\operatorname{diag}\left(B_{1}\right)>0$.

By Lemma 1.1, there is a positive vector $x$ such that

$$
T_{w, r} x=\lambda x
$$

Where $\lambda=\rho\left(T_{w, r}\right)$. Obviously, $\lambda=1$ is impossible, otherwise the matrix $H$ becomes singular. So we will mainly discuss two cases: $\lambda<1$ and $\lambda>1$.

Case 1: $\quad \lambda<1$. Since $\tilde{T}_{w, r} x-\lambda x=\tilde{T}_{w, r} x-T_{w, r} x$, we get

$$
\begin{aligned}
\tilde{T}_{w, r} x-T_{w, r} x & =\left[\begin{array}{cc}
-w S\left(I-B_{1}\right) & -w S D \\
w r C S\left(I-B_{1}\right) & w r C S D
\end{array}\right] x \\
& =\left[\begin{array}{cc}
S & 0 \\
-r C S & 0
\end{array}\right]\left[\begin{array}{cc}
-w\left(I-B_{1}\right) & -w D \\
0 & 0
\end{array}\right] x \\
& =\left[\begin{array}{cc}
S & 0 \\
-r C S & 0
\end{array}\right]\left(T_{w, r}-I\right) x \\
& =(\lambda-1)\left[\begin{array}{cc}
S & 0 \\
-r C S & 0
\end{array}\right] x .
\end{aligned}
$$

Since $S \geq 0$ and $S \neq 0$, then we get

$$
\left[\begin{array}{cc}
S & 0 \\
-r C S & 0
\end{array}\right] x \geq 0 \text { and }\left[\begin{array}{cc}
S & 0 \\
-r C S & 0
\end{array}\right] x \neq 0 .
$$

If $\lambda<1$, then $\tilde{T}_{w, r} x-T_{w, r} x \leq 0$ but not equal to zero vector. By Lemma 1.2, we get $\rho\left(\tilde{T}_{w, r}\right)<\rho\left(T_{w, r}\right)$. That is, 1) holds.

Similarly, 2) holds with $\lambda>1$, which completes the proof. $\square$

It is well known that when $w=r$, AOR iteration is reduced to SOR iteration. The following corollary is easily obtained.

Corollary 2.1 Let the coefficient matrix $H$ be irreducible, $B_{1} \geq 0$ with $\operatorname{diag}\left(B_{1}\right)>0, B_{2} \geq 0, C \leq 0$, $D \leq 0$ and $0<w<1$. Then

1) $\rho\left(\tilde{T}_{w}\right)<\rho\left(T_{w}\right)$, if $\rho\left(T_{w}\right)<1$;

2) $\rho\left(\tilde{T}_{w}\right)>\rho\left(T_{w}\right)$, if $\rho\left(T_{w}\right)>1$.

Next, we consider the following preconditioners. Let the matrix $\tilde{S}$ in (2.1) be defined by

$$
\tilde{S}=\left[\begin{array}{ll}
0 & 0 \\
S & 0
\end{array}\right] \text {. }
$$

There exist the following three forms for $S$, that is,

1) If $n-p<p$, then

$$
S=\left[\begin{array}{ccccccc}
-c_{11} & 0 & \cdots & 0 & 0 & \cdots & 0 \\
0 & -c_{22} & \cdots & 0 & 0 & \cdots & 0 \\
\vdots & \vdots & \ddots & \vdots & \vdots & \vdots & \vdots \\
-c_{n-p, 1} & 0 & \cdots & -c_{n-p, n-p} & 0 & \cdots & 0
\end{array}\right]_{(n-p) \times p}
$$

2) If $n-p=p$, then

$$
S=\left[\begin{array}{cccc}
-C_{11} & 0 & \cdots & 0 \\
0 & -c_{22} & \cdots & 0 \\
\vdots & \vdots & \ddots & \vdots \\
-c_{n-p, 1} & 0 & \cdots & -c_{n-p, n-p}
\end{array}\right]_{(n-p) \times p}
$$


3) If $n-p>p$, then

$$
S=\left[\begin{array}{cccc}
-c_{11} & 0 & \cdots & 0 \\
0 & -c_{22} & \cdots & 0 \\
\vdots & \vdots & \ddots & \vdots \\
-c_{p, 1} & 0 & \cdots & -c_{p, p} \\
\vdots & \vdots & \vdots & \vdots \\
0 & 0 & \cdots & 0
\end{array}\right]_{(n-p) \times p}
$$

Naturally, we assume that there at least exists a nonzero number in the elements of $S$. For the sake of simplicity, we assume that $n-p<p, \tilde{H}$ can be expressed as

$$
\tilde{H}=\left[\begin{array}{cc}
I-B_{1} & D \\
S\left(I-B_{1}\right)+C & I-B_{2}+S D
\end{array}\right]
$$

with

$$
\begin{aligned}
& S\left(I-B_{1}\right)+C \\
& =\left[\begin{array}{cccc}
c_{11} b_{11} & c_{12}+c_{11} b_{12} & \cdots & c_{1 p}+c_{11} b_{1 p} \\
c_{21}+c_{22} b_{21} & c_{22} b_{22} & \cdots & c_{2 p}+c_{22} b_{2 p} \\
\vdots & \vdots & \ddots & \vdots \\
d_{n-p, 1} & d_{n-p, 2} & \cdots & d_{n-p, p}
\end{array}\right]
\end{aligned}
$$

where

$$
\begin{aligned}
d_{n-p, 1} & =c_{n-p, 1} b_{11}+c_{n-p, n-p} b_{n-p, 1}, \\
d_{n-p, 2} & =c_{n-p, 2}+c_{n-p, 1} b_{12}+c_{n-p, n-p} b_{n-p, 2}, \\
& \ldots \\
d_{n-p, p} & =c_{n-p, p}+c_{n-p, 1} b_{1 p}+c_{n-p, n-p} b_{n-p, p} .
\end{aligned}
$$

The matrix $\tilde{H}$ is split as follows

$$
\tilde{H}=I-\left[\begin{array}{cc}
0 & 0 \\
-S\left(I-B_{1}\right)-C & 0
\end{array}\right]-\left[\begin{array}{cc}
B_{1} & -D \\
0 & B_{2}-S D
\end{array}\right] .
$$

Then the preconditioned AOR method for (2.1) is of the following form:

$$
x^{(i+1)}=\bar{T}_{w, r} x^{(i)}+w \bar{g},(w \neq 0), i=0,1, \cdots
$$

where

$$
\begin{gathered}
\bar{T}_{w, r}=(1-w) I+w J+w r K, \\
J=\left[\begin{array}{cc}
B_{1} & -D \\
-S\left(I-B_{1}\right)-C & B_{2}-S D
\end{array}\right], \\
K=\left[\begin{array}{cc}
0 & 0 \\
\left(S\left(I-B_{1}\right)+C\right)\left(I-B_{1}\right) & \left(S\left(I-B_{1}\right)+C\right) D
\end{array}\right],
\end{gathered}
$$

and

$$
\bar{g}=\left[\begin{array}{cc}
I & 0 \\
r\left(S\left(I-B_{1}\right)+C\right) & I
\end{array}\right] \bar{f} .
$$

Similarly, the following theorem and corollary are given by comparing the spectral radii of the iteration matrix $\bar{T}_{w, r}$ and the original iteration matrix $T_{w, r}$.

Theorem 2.2 Let the coefficient matrix $H$ be irreducible, $B_{1} \geq 0$ with $\operatorname{diag}\left(B_{1}\right)>0, B_{2} \geq 0, C \leq 0, D \leq 0$, $0<w \leq 1$ and $0 \leq r<1$. Then

1) $\rho\left(\bar{T}_{w, r}\right)<\rho\left(T_{w, r}\right)$, if $\rho\left(T_{w, r}\right)<1$;

2) $\rho\left(\bar{T}_{w, r}\right)>\rho\left(T_{w, r}\right)$, if $\rho\left(T_{w, r}\right)>1$.

Corollary 2.2 Let the coefficient matrix $H$ be irreducible, $B_{1} \geq 0$ with $\operatorname{diag}\left(B_{1}\right)>0, B_{2} \geq 0, C \leq 0, D \leq 0$, and $0<w<1$. Then

1) $\rho\left(\bar{T}_{w}\right)<\rho\left(T_{w}\right)$, if $\rho\left(T_{w}\right)<1$;

2) $\rho\left(\bar{T}_{w}\right)>\rho\left(T_{w}\right)$, if $\rho\left(T_{w}\right)>1$.

\section{A Numerical Example}

Now let us consider the following example to illustrate the results.

Example 3.1

$$
H=\left[\begin{array}{cc}
I-B_{1} & D \\
C & I-B_{2}
\end{array}\right],
$$

where

$$
\begin{gathered}
B_{1}=\left(b_{i j}^{(1)}\right)_{p \times p}, B_{2}=\left(b_{i j}^{(2)}\right)_{(n-p) \times(n-p)}, \\
C=\left(c_{i j}\right)_{(n-p) \times p}, \text { and } D=\left(d_{i j}\right)_{p \times(n-p)}
\end{gathered}
$$

with

$$
\begin{aligned}
b_{i i}^{(1)}= & \frac{1}{50}, i=1,2, \cdots, p, \\
b_{i j}^{(1)}= & \frac{1}{40}-\frac{1}{40 j+i}, i<j, \\
& (i=1, \cdots, p-1, j=2, \cdots, p)
\end{aligned}
$$$$
b_{i j}^{(1)}=\frac{1}{40}-\frac{1}{40(i-j+1)+i}, i>j,
$$$$
(i=2, \cdots, p, j=1,2, \cdots, p-1)
$$$$
b_{i i}^{(2)}=\frac{1}{40}, i=1,2, \cdots, n-p,
$$$$
b_{i j}^{(2)}=\frac{1}{40}-\frac{1}{40(p+j)+p+i}, i<j,
$$$$
(i=1, \cdots, n-p+1, j=2, \cdots, n-p)
$$

$$
\begin{aligned}
b_{i j}^{(2)}= & \frac{1}{40}-\frac{1}{40(i-j+1)+p+i}, i>j, \\
& (i=2, \cdots, n-p, j=2, \cdots, n-p-1)
\end{aligned}
$$


Table 1. The spectral radii of the AOR and preconditioned AOR iteration matrix.

\begin{tabular}{ccccccc}
\hline$n$ & $p$ & $w$ & $r$ & $\rho\left(T_{w, r}\right)$ & $\rho\left(\tilde{T}_{w, r}\right)$ & $\rho\left(\bar{T}_{w, r}\right)$ \\
\hline 5 & 3 & 0.95 & 0.8 & 0.1153 & 0.1081 & 0.1088 \\
10 & 6 & 0.9 & 0.5 & 0.2591 & 0.2513 & 0.2524 \\
15 & 5 & 0.9 & 0.85 & 0.3379 & 0.3351 & 0.3358 \\
20 & 10 & 0.75 & 0.6 & 0.5422 & 0.5376 & 0.5379 \\
30 & 18 & 0.8 & 0.75 & 0.7005 & 0.6960 & 0.6982 \\
40 & 30 & 0.5 & 0.3 & 0.9582 & 0.9575 & 0.9579 \\
50 & 25 & 0.9 & 0.5 & 1.1774 & 1.1796 & 1.1793 \\
60 & 20 & 0.8 & 0.5 & 1.3923 & 1.3957 & 1.3948 \\
\hline
\end{tabular}

Table 2. The spectral radii of the SOR and preconditioned SOR iteration matrix.

\begin{tabular}{cccccc}
\hline$n$ & $p$ & $w=r$ & $\rho\left(T_{w, r}\right)$ & $\rho\left(\tilde{T}_{w, r}\right)$ & $\rho\left(\bar{T}_{w, r}\right)$ \\
\hline 5 & 3 & 0.95 & 0.1079 & 0.1003 & 0.1038 \\
10 & 5 & 0.9 & 0.2321 & 0.2261 & 0.2276 \\
15 & 5 & 0.8 & 0.4148 & 0.4123 & 0.4127 \\
20 & 15 & 0.75 & 0.5427 & 0.5345 & 0.5404 \\
30 & 18 & 0.65 & 0.7622 & 0.7587 & 0.7602 \\
40 & 30 & 0.6 & 0.9467 & 0.9457 & 0.9464 \\
50 & 25 & 0.8 & 1.1749 & 1.1773 & 1.1766 \\
60 & 20 & 0.5 & 1.2452 & 1.2473 & 1.2468 \\
\hline
\end{tabular}

$$
\begin{aligned}
c_{i j}= & \frac{1}{40(p+i-j+1)+p+i}-\frac{1}{40}, \\
& (i=1, \cdots, n-p, j=1,2, \cdots, p) \\
d_{i j}= & \frac{1}{40(p+j)+i}-\frac{1}{40}, \\
& (i=1, \cdots, p, j=1,2, \cdots, n-p)
\end{aligned}
$$

Tables 1, 2 display the spectral radii of the corresponding iteration matrix with different parameters $w$, $r$ and $p$. These calculations are performed using Matlab 7.1.

Obviously, from Table 1, it easy to known that
$\rho\left(\tilde{T}_{w, r}\right)<\rho\left(T_{w, r}\right)<1$ and $\rho\left(\bar{T}_{w, r}\right)<\rho\left(T_{w, r}\right)<1$. That is, these are in concord with Theorem 2.1 and 2.2.

From Table 2, it is easy to know that $\rho\left(\tilde{T}_{w}\right)<\rho\left(T_{w}\right)$ and $\rho\left(\bar{T}_{w}\right)<\rho\left(T_{w}\right)$ when $\rho\left(T_{w}\right)<1$. That is, these are in concord with Corollary 2.1 and 2.2 .

\section{Acknowledgements}

This research was supported by NSFC (11026040, 11026083).

\section{References}

[1] S.-L. Wu and T.-Z. Huang, "A Modified AOR-Type Iterative Method for L-Matrix Linear Systems," Australian \& New Zealand Industrial and Applied Mathematics Journal, Vol. 49, 2007, pp. 281-292.

[2] J.-Y. Yuan, "Iterative Methods for Generalized Least Squares Problems," Ph.D. Thesis, IMPA, Rio de Janeiro, Brazil, 1993.

[3] J.-Y. Yuan, "Numerical Methods for Generalized Least Squares Problems," Journal of Computational and Applied Mathematic, Vol. 66, No. 1-2, 1996, pp. 571-584. doi:10.1016/0377-0427(95)00167-0

[4] J.-Y. Yuan and A. N. Iusem, "SOR-Type Methods for Generalized Least Squares Problems," Acta Mathematicae Applicatae Sinica, Vol. 16, 2000, pp. 130-139. doi:10.1007/BF02677673

[5] J.-Y. Yuan and X.-Q. Jin, "Convergence of the Generalized AOR Method," Applied Mathematics and Computation, Vol. 99, No. 1, 1999, pp. 35-46. doi:10.1016/S0096-3003(97)10175-8

[6] R. S. Varga, "Matrix Iterative Analysis," Springer Series in Computational Mathematics: 27, Springer-Verlag, Berlin, 2000.

[7] D. M. Young, "Iterative Solution of Large Linear Systems," Academic Press, New York, 1971.

[8] A. Hadjidimos, "Accelerated over Relaxtion Method," Mathematics of Computation, Vol. 32, No. 141, 1978, pp. 149-157. doi:10.1090/S0025-5718-1978-0483340-6

[9] X.-X. Zhou, Y.-Z. Song, L. Wang and Q.-S. Liu, "Preconditioned GAOR Methods for Solving Weighted Linear Least Squares Problems," Journal of Computational and Applied Mathematics, Vol. 224, No. 1, 2009, pp. 242-249. doi:10.1016/j.cam.2008.04.034

[10] A. Berman and R. J. Plemmons, "Nonnegative Matrices in the Mathematics Sciences," SIAM, Philadelphia, 1994. 